\title{
An Automatic SAR Image Segmentation Framework by Multi-objective Clustering and Artificial Immune Learning
}

\author{
Dongdong Yang ${ }^{1, *}$, Xiaowei Zhang ${ }^{2}$, Lintao $\mathrm{Lv}^{3}$ and Wenzhun Huang ${ }^{4}$ \\ ${ }^{1}$ Information Engineer Department, Xijing University, Xi’an, China; School of Computer Science Engineering, Xi’an University \\ of Technology, Xi'an, China \\ ${ }^{2}$ Information Engineer Department, Xijing University, Xi’an, China \\ ${ }^{3}$ Information Engineer Department, Xijing University, Xi'an, China; School of Computer Science Engineering, Xi'an University \\ of Technology, Xi'an, China \\ ${ }^{4}$ Information Engineer Department, Xijing University, Xi’an, China \\ ${ }^{*}$ Corresponding author
}

\begin{abstract}
Though several algorithms inspired by theoretical immunology have been applied to the domain of pattern classification, little focus has been placed on the issues that simultaneously optimize more than one objective-functions. Here, an efficient multi-objective automatic segmentation framework (MASF) is formulated and applied to SAR image unsupervised classification. In the framework, four important issues are presented: 1) two reasonable image preprocessing techniques are discussed at the initial stage; 2)then, an efficient immune multiobjective optimization algorithm is proposed; 3) besides, a locusbased adjacency representation in individual encoding is introduced; 4) two very simple, but very efficient conflicting clustering validity indices are incorporated into the framework and simultaneously optimized. Both simulated data and real images are used to quantitatively validate its effectiveness. In addition, four other state-of-the-art image segmentation methods are employed for comparison. Experimental results show that the proposed framework is efficient and effective for SAR image segmentation.
\end{abstract}

Keywords-evolutionary computation; artificial immune system; SAR image segmentation; multi-objective optimization; automatic clustering; watershed transformation

\section{INTRODUCTION}

Synthetic Aperture Radar (SAR) can obtain images at high resolution from broad areas of terrain in all weather, day and night and long distance conditions. The purpose of SAR image segmentation is to partition an image into regions of different characteristics, that is to say, partitioning a given image samples into groups such that the samples in a group are more similar to each other than samples in different groups. The main difficulties for SAR image segmentation are the complicated and vast image samples, and speckle noise introduced in the process of imaging. For a SAR image with size of 256x256, there are 65536 samples in it, which can bring big technical challenge to traditional clustering methodologies for real time and accuracy requirement. In addition, SAR image is generated by sending electromagnetic waves from a moving platform towards the target surface, and coherently integrating the returned backscattered energy. Inevitably, the coherent integration introduces the speckle noise into the process of imaging. The existence of noise deteriorates the quality of SAR images and conceals important details, which leads to the loss of interesting objectives.

SAR image segmentation has been the subject of widely research since the invention of SAR imaging. Up to now, a multitude of methods have been proposed, which can be classified into clustering-based methods [1], graph-partitioning methods [2], morphologic methods [3], and model-based methods [4]. The paper mainly concerns the methods of clustering-based SAR image segmentation. To the best of our knowledge, k-means is the most cited and widely used method for data clustering and image classification. The algorithm is easy to implement and it gives reasonable results in most cases. Unfortunately, the algorithm makes only local changes to the initial partition and it may sink into local optima at the early iteration stage. The quality of the final clustering is therefore highly dependent on the initialization, especially for the case where there are many true categories in the classification task. Recently, a group of spectral clustering algorithms were proposed and have shown encouraging experimental results on a number of clustering problems with spherical shapes and some linear non-separable shapes. Most of spectral clustering algorithms treat image segmentation as a graph partitioning problem and some global criterions by eigenvalue decomposition are then made to divide all samples into disjoint sets. However, the weak points of spectral clustering algorithms lie in their expensive computational time and hard setting of scaling parameter in the Gaussian radial basis function. In a paper by Zhang [5], she provided a novel spectral clustering ensemble algorithm (SCE) to avoid the selection of the appropriate scaling parameter. Furthermore, a part of randomly data points sampled from all image pixels is used in each baselevel learner of the ensemble, which has been shown to obviously reduce the complexity of the original algorithms. Besides, evolutionary computation (EC) inspired by biology evolution provides another new idea for clustering analysis. Bandyopadhyay and Maulik have proposed a frequently cited 
evolutionary clustering algorithm, called variable string length genetic algorithm (VGA) [6]. The cluster centers with variable length of the chromosome in VGA are encoded in real values; therefore, it can automatically discover the number of the clusters. In another paper, Liu et al. [7] presented an improved variant of the clonal selection algorithm by gene transposition (GTCSA) and applied to complicate automatic clustering. The gene transposition is used to change the length of antibody string for finding the optimal number of clustering automatically. They have shown that GTCSA performs better than VGA over nine complicated multi-class test problems under their study. However, the common shortcomings of VGA and GTCSA are their large computational load and implicit spherical-shapes encoding scheme in input space. Consequently, it seems to be inappropriate for them to partition data sets with large samples and non-spherical shapes.

In this study, we present an automatic SAR image segmentation Framework by multi-objective clustering and artificial Immune Learning (MASF). Multi-objective optimization (MO), also known as multi-criteria optimization, is the process of simultaneously optimizing two or more conflicting objectives subject to certain constraints. Evolutionary computation is extremely suitable to solve $\mathrm{MO}$ problems because of its population parallel searching strategy. Usually, a set of optimal tradeoff solutions known as the Pareto-optimal solutions in MO can be obtained, and then, the optimal or user wanted solutions can be selected from the tradeoff solutions set. Furthermore, the artificial immune system simulates highly evolved, parallel, and distributed adaptive the characteristics of human immune system to develop computational tools for dealing with science and engineering problems. Recently, AIS-based algorithms have begun to emerge as problem solvers in pattern classification and image segmentation. We have shown that AIS based multi-objective optimization is an efficient and competitive methodology in current evolutionary computation.

The main new contributions of this study and their rationalities are discussed as follows. Firstly, mass samples and speckle noises in SAR images are two crucial problems in devising an efficient and effective SAR segmentation framework. Mostly, evolutionary computation with population iteration on the level of pixels is very time consuming, even for small SAR images of moderate resolution. With this in mind, a preprocessing stage is formulated in the framework and characterized by firstly removing speckle noise and then oversegmenting the original image into reasonable number of local regions. Secondly, we introduce an efficient and robust AISbased two-objective optimization with uniform clone, adaptive selection by online found nondominated solutions, and a diversity maintenance technique by K-nearest neighbor list (where $\mathrm{K}$ is the number of objectives) for subsequent fine partition. Besides, a novel individual encoding scheme by locus-based adjacency graph with the ability of automatic discovering cluster numbers is introduced here. Most importantly, this encoding strategy doesn't bias toward any sample models, that is to say, it can supply equal opportunities between different objectives in MO. Additionally, the clustering indexes, also called objective-functions, are discussed here, and two enhanced ones are incorporated into the framework and simultaneously optimized. To validate the effectiveness of the framework, four other state-of-the-art image segmentation methods are employed for comparison in partitioning two simulated SAR images and two very complicated real ones. The experimental results demonstrated the proposed approach has achieved the better classification performance than other four algorithms and has remarkably reduced time complexity of VGA and GTCSA.

\section{Definitions of MUlti-OBJEctive Optimization}

If more than one objective functions in a problem are required to be optimized simultaneously, it consists on a multiobjective optimization. The basic definition of it could be formulated as follows.

$$
\min \boldsymbol{F}(\boldsymbol{x})=\left(f_{1}(\boldsymbol{x}), f_{2}(x), \mathrm{L}, f_{K}(x)\right)^{T}
$$

Where $\mathrm{x}$, subject to $\boldsymbol{x} \in \Omega$, is a decision variable vector and $\Omega$ is the search space. $\boldsymbol{F}: \boldsymbol{x} \rightarrow R^{K}$ is the map of decision variable space to the space of $\mathrm{K}$ real valued objectives. The objectives in multi-objective optimization usually conflict with each other and no single solution can optimize all the objectives simultaneously. Therefore, a set of optimal tradeoff solutions known as the Pareto-optimal solutions can be obtained. The optimal or user wanted solutions of clustering tasks can be selected from the tradeoff Pareto-optimal solutions.

A solution $\boldsymbol{u}=\left(u_{1}, u_{2}, \mathrm{~L}, u_{K}\right)$ is said to dominate another solution $\boldsymbol{v}=\left(v_{1}, v_{2}, \mathrm{~L}, v_{K}\right)$ (denoted by $u \mathbf{p} v$ ) if and only if $\mathrm{u}$ is partially less than $\mathrm{v}$, which can be defined by the following expression:

$$
\forall i \in\{1, \mathrm{~L}, K\}, u_{i} \leq v_{i} \wedge \exists j \in\{1, \mathrm{~L}, K\}: u_{j}<v_{j}
$$

Where $\wedge$ is a logic symbol, which mean two concurrent terms. Furthermore, we say that a solution $\boldsymbol{x}^{*} \in \Omega$ is Paretooptimal or a non-dominated solution when any solution, $\boldsymbol{x} \in \Omega$ does not exist such that $\boldsymbol{x} \mathrm{p} \boldsymbol{x}^{*}$. The non-dominated solutions are usually better than the dominated solutions in the real-world applications.

In this study, we employ the two modified clustering validity indices of Dev and Conn, called MDev and MConn. The definitions of them can be described as follows. If there are $\mathrm{m}$ solutions in the current population, the values of the two indices of $t$-th solution can be defined by equation (3) and (4).

$$
\begin{aligned}
& \operatorname{Dev}(t)=\sum_{i=1, C_{i} \in C}^{k(t)} \sum_{j \in C_{i}} d\left(j, m_{i}\right), m_{i}=\frac{1}{\left|C_{i}\right|} \sum_{x_{j} \in C_{i}} x_{j} \\
& \operatorname{MDev}(t)=\text { ウ }[k(t)] *[\operatorname{Dev}(t)]
\end{aligned}
$$




$$
\begin{aligned}
& \operatorname{MConn}(t)=\sum_{i=1}^{n} \sum_{j=1}^{L} v\left(x_{i, j}\right), \\
& \text { where } v\left(x_{i, j}\right)=\left\{\begin{array}{c}
d\left(x_{i}, x_{j}\right) / j,: \exists C_{m}: x_{i} \in C_{m} \wedge x_{j} \in C_{m} \\
0, \quad \text { otherwise }
\end{array}\right.
\end{aligned}
$$

where $\mathrm{k}$ is the number of clusters of $\mathrm{t}$-th solution, $x_{i}$ and $x_{j}$ are two datum in the cluster subset of $C_{i}$ and $C_{m}$ respectively. $¥[\mathrm{~L}]$ denotes the normalization of the data items in the bracket into the range of $[0,1]$. It can be seen that Dev-index is defined by global with-cluster scatter, which can measure the total variations of the partition. However, the extreme case of Dev-index is zero when all the data samples are their own cluster centers respectively. Thus, Dev-index is strongly biased toward the partitions with high number of clusters, which is not the beneficial to automatic clustering. To this end, it is multiplied by the normalized cluster numbers to build the tradeoff between the two items. In additionally, MConn-index is formulated by dividing Euclidian distance of datum $\mathrm{i}$ to their $\mathrm{j}$-th nearest neighbor by gradually decreasing penalty factor $j \in(1,2, L, L)$. Thus, the nearer the neighbors of datum i close to it, the less the values of the penalty. Therefore, it is beneficial to measure the cluster connectedness. The original definition of Dev-index just has the penalty, other than the item of distance of datum to their $L$ nearest neighbor. It doesn't have clear meanings to measure each datum to their neighbors.

\section{PREPARE YOUR PAPER BEFORE STYLING}

In this study, we want to present an efficient and effective two-objective automatic SAR image segmentation framework. The basic procedure of the SAR image segmentation framework is illustrated in Figure 1. Clearly, it can be divided into two stages. The first stage is the preprocessing operations, including speckle noise removing and coarse segmentation, and the second stage consist of an efficient AIS multi-objective clustering algorithm, which can implement the fine segmentation on the initial partitioning results of the first stage and final results presentation.

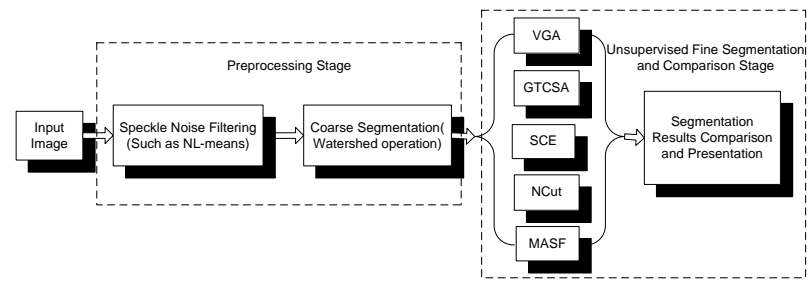

FIGURE I. BASIC PROCEDURE OF THE SAR IMAGE SEGMENTATION FRAMEWORK BY IMMUNE MULTIOBJECTIVE OPTIMIZATION AND OTHER FOUR FAMOUS IMAGE PARTITIONING ALGORITHMS

\section{A. Preprocessing Techniques of the First Stage}

1) Non-local Means Algorithm for Image Denoising:

Non-local means makes the best use of redundancy in images, which means that every small patch in an image have many similar patches in the same image [3]. As a result, the speckle randomly distributed in the image can be suppressed and the fine structure, details and textures can be preserved. If $I$ is an SAR image with noises, $v(i)$ is the observed image and $N L(i)$ is the noise removed image by non-local means, the denoising operator can be defined as the weighted average of the pixels in the original image.

$$
N L(i)=\sum_{j \in I} w(i, j) v(j)
$$

The weights in equation (5) measures the similarity of pixel $\mathrm{i}$ and their neighborhoods $N B_{i}$ defined in a square-block of fixed size around pixel i. $v\left(N B_{i}\right)$ is the vector of neighborhood pixel around $i$, and defined by $v\left(N B_{i}\right)=(v(j)), j \in N B_{i}$. The weights can be calculated in the following equation.

$$
w(i, j)=\frac{1}{\operatorname{Nor}(i)} e^{-\frac{\left\|v\left(N B_{i}\right)-v\left(N B_{j}\right)\right\|_{2, a}^{2}}{h^{2}}}, \operatorname{Nor}(i)=\sum_{j} e^{-\frac{\left\|v\left(N B_{i}\right)-v\left(N B_{j}\right)\right\|_{2, a}^{2}}{h^{2}}}
$$

where $\operatorname{Nor}(i)$ is a normalizing factor, a is the standard deviation of the Gaussian kernel, and h controls the decays of the exponential function. If an SAR image has $M$ pixels, $M$ weights are required to be calculated for each pixel, which is very vast computational load for this denoising method. Usually, we can restrict the neighborhood size of each pixel using the assumption that the similarity between pixels decreases when they are located far from each other.

\section{2) Watershed Raw Segmentation:}

The watershed transformation is to partition SAR image into disjoint small regions, such that each region is homogenous with respect to grey value [25]. The definition of watershed transformation is as follows:

$$
W T(I)=\frac{1}{N} \sum_{i=1}^{N}\left[I \oplus B_{i}-\left(I \Theta B_{i}\right) \Theta B_{i-1}\right]
$$

where $\oplus$ and $\Theta$ denote dilation and erosion operation in mathematical morphology respectively, and $B_{i}$ is called structural window with size $(2 i-1) \times(2 i-1)$, and $I$ is the original image. This transform will produce an important oversegmentation due to noise or local irregularities in the image, which is far from being an easy task to implement suitable watershed transformation. Fortunately, the over-segmented regions supply enough samples for subsequent fine partitioning in the second stage in Figure 2. Furthermore, the shortcoming of watershed transformation in this study can be changed to merit for the subsequent fine segmentation algorithms. A gradient-based watershed transformation and morphological operator are employed here. Specifically, the sizes of dilation and erosion operation are 3x3 window and about 1000 "superpixels" are obtained in an image with 256x256. 


\section{B. Two-objective Automatic SAR Image Segmentation}

In this section, the human immune system inspired multiobjective metaphor will be presented. It is worthwhile to note that we have successively applied AIS into multi-objective optimization and proposed an outstanding multi-objective optimization using AIS [28], called NNIA2. It possessed the characteristics of enhanced robustness, adaptability, and the ability of diversity maintaining. The multi-objective problems solved in the literature [28] have continuous searching space in the variable domain. However, the SAR image segmentation is a kind of the discrete problem. Consequently, some efficient strategies may be not suitable to deal with such problem. For example, the adaptive ranks clone in NNIA2 will allocate much more computational budget to the individuals locating at the less-crowded regions of the different trade-off front, but there is no continuity of the discrete problem, i.e. there may be not promising solutions between the current solutions. Thus, it is unreasonable and blind for computational resource assignment in NNIA2, which isn't beneficial to the success of the search and optimization processes. In this study, we proposed an improved version of NNIA2 for SAR image segmentation. It possesses the characteristics of uniform clone, adaptive selection by online nondominated solutions and dynamic deletion in diversity maintenance. The procedure is described in Table 1.

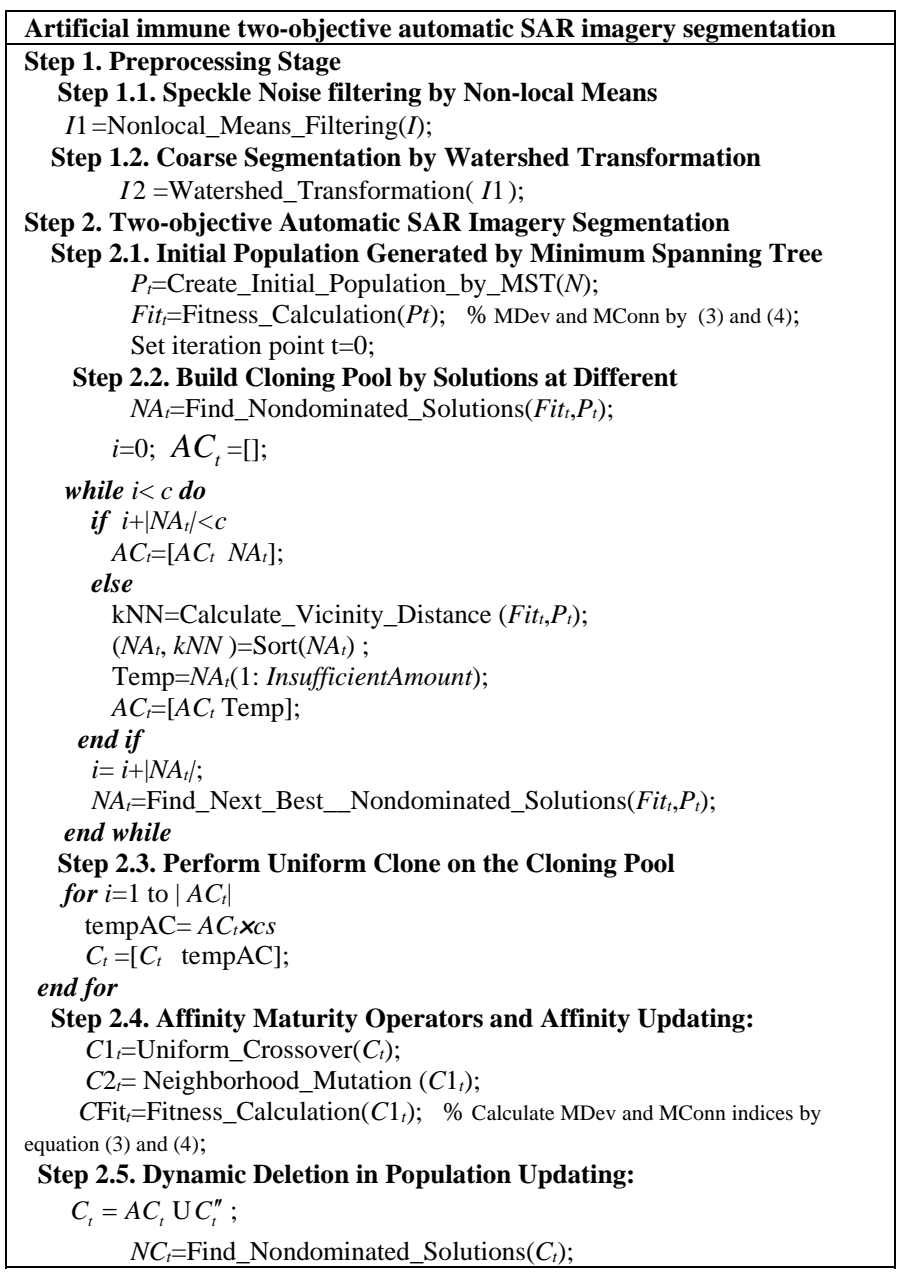

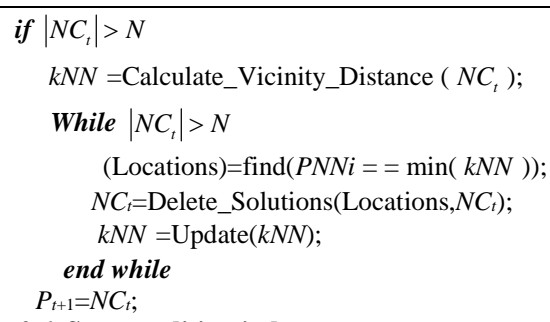

Note that diversity maintenance and selection pressure are two critical issues in both single-objective and multi-objective optimization, which is often crucial to the success of the search and optimization processes. Here, in order to keep the balance of the searching process, the population updating is based on the number of online discovered nondominated solutions. We can see that the population size $\mathrm{N}$ and the cloning scale $\mathrm{c}$ are two crucial thresholds for $P_{t+1}$ in Step 2.5 in Table 1. Intuitively and obviously, if the current nondominated solutions are larger than $\mathrm{N}$, the diversity of population can be held by nondominated solutions. Instead, once it is less than c, the quality of diversity of whole population can't be measured by nondominated solutions. The solutions before cloning operator in current cloning pool are used to update the next population, which is one step pause in searching process for diversity accumulation. Besides, we have successively used the online discovered number of nondominated solutions as feedback to devise the adaptive searching system in complicated multiobjective test problems. We believe that the adaptive and robust searching scheme also can perform effective in the SAR image segmentation framework.

\section{EXPERIMENTAL STUDY}

How about the efficiency of the above techniques in the framework is? Here, four state-of-the-art image partitioning algorithms are employed to investigate the relative performance of MASF. The first two methods are VGA [6] and GTCSA [7]. They are both the type of clustering algorithms devised in evolutionary computation and can automatically find the optimal cluster number. The source codes of them are provided by Prof. Liu [7]. Additionally, the next algorithm is the current popular graph partitioning method, called NCut The procedure could be downloaded from website (http://www.seas.upenn.edu/ timothee/software/ncut/ncut.html). The last method for comparing is a recently proposed SAR image segmentation algorithm, called SCE [5]. The unsupervised ensemble is combined with spectral clustering to fill up its shortcomings. The source code of SCE is supplied by the original writer, Prof. Zhang.

\section{A. Experimental Results Presentation and Discussion}

The experimental results of the five algorithms on segmenting the artificial SAR images and real ones will be presented here. Note that there are ten classes in gray levels in the first synthetic SAR images and the image size is about 256x256. Besides, the second SAR image with the size of $512 \times 512$ has eight categories. Both of the two SAR images are to follow the multiplicative Goodman's speckle noise model [8]. It can be easily obtained that these two SAR images have 65536 and 262144 pixels respectively. Therefore, the vast data points 
mostly bring trouble for the current clustering algorithms on the level of pixels. It is very necessary to pre-segment the huge mass of pixels into local coherent regions. In this study, these two artificial images are called AI1 and AI2 for short in the following paragraphs.

Figures 2 show the segmentation results of the synthetic SAR images with ten categories using the five algorithms, respectively. In both of two figures, we can see that VGA, GTCSA and MASF have obtained the better segmentation results in visual inspection among the five algorithms. Some patches in Figure 2(d), Figure 2(e) obtained by SCE and NCut are misclassified.

Figure 3(a) shows the original SAR sub-image of Nördlinger Ries in the Swabian Jura, German, which can supply the study of microstructures of farm crops and analyses of surface formations to improve agricultural utilization. The original of the SAR image can be downloaded freely from the German Aerospace Center. The land-covers in the image can be divided into five classes: four types of crops and some buildings in the village at the low left of the image. In Figure 6, we can see that VGA and GTCSA still over-segment the image into many redundant classes. It is intuitive that the speckle noise can't be removed completely by non-local means because it still can't be estimated exactly by any model up to now. Consequently, the subsequent partitioning algorithms in the second stage of the framework remain to be confronted with the trouble induced by the residual noise. Therefore, these may be another reason that why VGA and GTCSA classify the real SAR images into excessive categories. Keep on considering results of Figure 6, it seems that SCE and MASF demonstrated the better two partitions on the SAR image among the five algorithms.

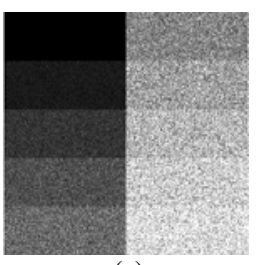

(a)

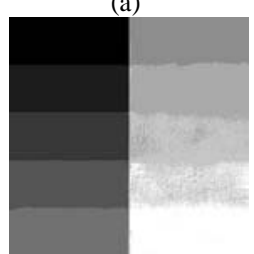

(d)
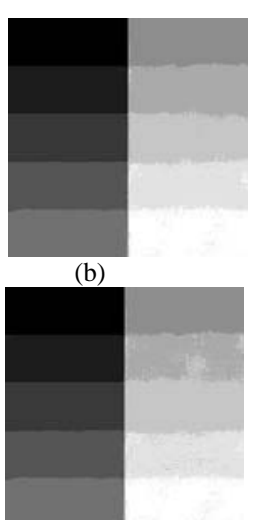

(e)
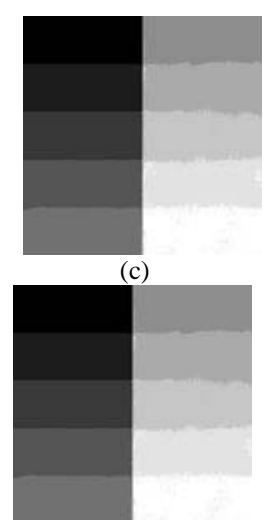

(f)
FIGURE II. THE SEGMENTATION RESULTS OF THE SYNTHESIZED SAR IMAGE WITH TEN CATEGORIES. (A) THE ARTIFICIAL SAR IMAGE (256 X 256 PIXELS), CALLED AI1; (B)-(F) SEGMENTATION RESULTS BY VGA(10), GTCSA(10), SCE(10), NCUT(10), AND MASF(10) RESPECTIVELY (THE NUMBER IN

THE BRACKET IS THE DISCOVERED NUMBER OF CATEGORIES)

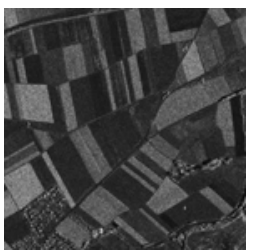

(a)

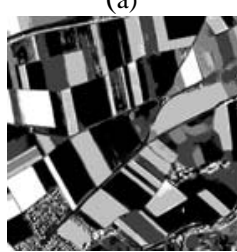

(d)

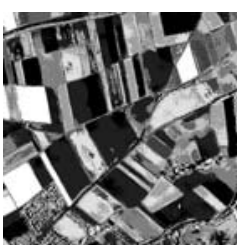

(b)

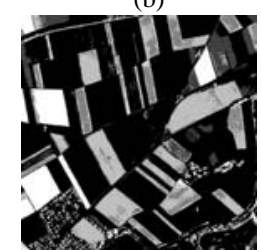

(e)

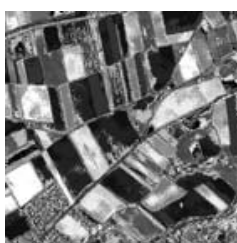

(c)

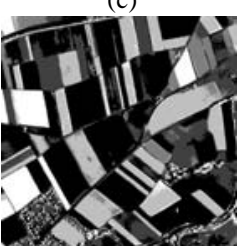

(f)
FIGURE III. THE SEGMENTATION RESULTS OF THE REAL SAR IMAGE WITH FIVE CATEGORIES. (A) THE ORIGINAL REAL SAR IMAGE (512 X 512 PIXELS), CALLED SAR2; (B)-(F) SEGMENTATION RESULTS BY VGA(9), GTCSA(16), SCE(5), NCUT(5), AND MASF(5) RESPECTIVELY (THE NUMBER IN THE BRACKET IS THE DISCOVERED NUMBER OF CATEGORIES)

It is always a dream for engineer in remote sensing community to build an efficient and effective SAR image segmentation framework. In this study, we proposed a twoobjective automatic segmentation framework. The procedure and rationality of the framework are discussed. Importantly, an efficient immune multi-objective optimization algorithm with uniform clone, dynamic deletion in diversity maintenance, and adaptive selection by online discovered non-dominated solutions is proposed. Beside, the locus-based adjacency representation in individual encoding and two very simple, but very efficient clustering validity indices are incorporated into the framework. The proposed methodology has been shown to outperform four other state-of-the-art SAR image partitioning methods. Furthermore, it achieved the effectiveness at the expense of a reasonable computational effort.

\section{ACKNOWLEDGMENT}

This work was supported by the National Natural Science Foundation of China (Grant Nos, 61273271, and 61403304), the Project of Industrial Technology of Shaanxi Province, China (Grant No.2016GY-141) , and New Science Star of Shaanxi Province, China (Grant No.2017KJXX-30)..

\section{REFERENCES}

[1] A.K. Jain, R.P. Duin, J.C. Mao, "Statistical pattern recognition: A review”, IEEE Trans. Pattern Analysis and Machine Intelligence, vol. 22, no.1, pp.4-37, 2000.

[2] J. Shi, J. Malik, "Normalized cuts and image segmentation”, IEEE Trans. on Pattern Analysis and Machine Intelligence, vol.22, no.8, pp.888-905, 2000.

[3] M.G. Gong, L.Z.Su, M. Jia, W.S. Chen, "Fuzzy clustering with modified MRF energy function for change detection in synthetic aperture radar images”, IEEE Trans. on Fuzzy Systems, vol. 22, no. 1, pp.98-109, 2014.

[4] F. Wang, Y.Wu, M. Li, P. Zhang, Q.J. Zhang, "Adaptive hybrid conditional random field model for SAR image segmentation”, IEEE Trans. on Geoscience and Remote Sensing, vol.55, no.1, pp: 537 - 550, 2017.

[5] X.R. Zhang, L.C. Jiao, F. Liu, L.F. Bo, M.G. Gong, "Spectral clustering ensemble applied to SAR image segmentation”, IEEE Trans. Geoscience and Remote Sensing,, vol.46, no.7, pp: 2126 - 2136, 2017. 
[6] S. Bandyopadhyay, U. Maulik, "Nonparameter genetic clustering: comparison of validity indices", IEEE Trans.Systems, Man, and Cybernetics-Part C, vol.31,no.1,pp:120-125,2001.

[7] R.C. Liu, Z.C. shen, L.C. Jiao, "Gene transposon based clonal selection algorithm for clustering", Proceedings of the 11th Annual conference on Genetic and evolutionary computation, GECCO’09, Montréal, Québec, Canada, pp:1251-1258, 2009.

[8] Y.T. Wang, T. L. Ainsworth, J.S. Lee, "Application of mixture regression for improved polarimetric SAR speckle filtering”, IEEE Trans. on Geoscience and Remote Sensing, vol.55, no.1, pp:453-467, 2017. 